\title{
Communicating the Changing Role of a Nurse in an Epidemic: The Example of the MERS-CoV Outbreak in Saudi Arabia
}

\begin{abstract}
Context: In the event of outbreaks of infectious diseases, the role of the nurse changes to adapt to the needs of the patient, their families, and the hospital. Articulating the changes in the nurses' role is helpful for communicating a plan to decrease the spread of disease and for implementing improved policies, procedures, and supplies.
\end{abstract}

Objective: This article outlines the reflections made by a team of nurses on how roles changed during the recent Middle East Respiratory Syndrome Corona Virus (MERS-CoV) epidemic.

Design: A survey was used to ask nurses about their roles and how they changed as they worked with MERS-CoV patients during the height of the epidemic in Saudi Arabia.

Participants: The nurse researchers interviewed 9 critical care nurses about their past experiences with MERS-CoV patients.

Main outcome: The nurses identified areas that need to be improved in preparation for future epidemics.

Conclusion: The importance of communication during these complex outbreaks is considered. Much of the experience gleaned from this incident can be used to guide future practice in epidemics of MERS-CoV and other infectious diseases.

Keywords: Coronavirus; Epidemic; Intercultural communication; MERS-CoV; Nurses; Role of the nurse; Saudi Arabia

\section{Bridget Stirling ${ }^{1}$,} Jillian Hatcher ${ }^{2}$ and Jennie Harmston ${ }^{3}$

1 College of Nursing, University of Calgary in Qatar, Doha, Qatar

2 King Faisal Research and Specialist Hospital, Riyadh, Saudi Arabia

3 Cornwall Partnership NHS Foundation Trust, UK

\section{Corresponding author: Bridget Stirling}

झ bridget.stirling@ucalgary.edu.qa

College of Nursing, University of Calgary in Qatar, Doha, Qatar.

Tel: 7804926434

Citation: Stirling B, Hatcher J, Harmston J. Communicating the Changing Role of a Nurse in an Epidemic: The Example of the MERS-CoV Outbreak in Saudi Arabia. J Healthc Commun. 2017, 2:3.

\section{Introduction}

\section{Healthcare communication}

Effective communication is the key to successful healthcare team. Nurses communicate with physicians, nutritionists, physiotherapists, patients, families and many others. Communication takes place through body language, touch, listening, writing, reading and if necessary by speaking. New technologies such as electronic medical records and innovations such as patient bedside whiteboards have encouraged information exchange between nurses and others. However, even under the best and most stable situations, communication can breakdown.

\section{Nursing in Saudi Arabia}

Two barriers to effective communication in Saudi Arabia are the way that nurses are perceived by society and the healthcare team, and the multi-national backgrounds of the nurses themselves. While nurses are generally respected and trusted in most of the world [1,2], this is not necessarily true in all societies. In Saudi Arabia, nursing is viewed as a lowly profession [3]. The Middle East has fewer nurses per capita than other regions; with Saudi Arabia having almost half as many per capita as Canada [4]. Having fewer numbers of nurses, most of whom have English as their second language can cause nurses to become overburdened and to have communication difficulties. Nurses' voices are often not heard during patient care discussions. Oftentimes, 
nurses' opinions are not listened to, and while carrying a great deal of responsibility, are given very little authority.

The vast majority of the nurses in Saudi Arabia (71\%) are foreign $[5,6]$. This large and growing group of International professionals are imported to provide nearly all essential nursing services and funded by Saudi Arabia's economic wealth from oil. While the language of the medical system in Saudi Arabia is English, most of the nurses and nearly all of the patients do not have English as their first language. Under normal situations, nurses' roles are not always clear. In the case of a deadly epidemic, they may become even more uncertain. Communication therefore of changing roles and expectations becomes more important during these times. Table 1 outlines some important information about MERS-CoV [7].

This paper outlines a summary of the responses given by a group of foreign Critical Care nurses, who worked during the MERS-CoV in Riyadh, Saudi Arabia in May, 2014. The lessons learned in this paper could be applied during any droplet or airborne epidemics that nurses face throughout the world.

\section{Epidemiology of MERS-CoV}

As of April 4, 2017, MERS-CoV is confirmed to have infected 1,936 people in more than 25 countries worldwide, and resulted in 690 deaths [8]. This results in a case-fatality rate of about $36 \%$. Clinically, MERS-CoV is difficult to detect for the first few days after infection. To begin with, symptoms range from either mild respiratory symptoms or the patient is asymptomatic. As the infection progresses, the patient could experience severe acute respiratory disease, gastrointestinal symptoms, kidney shutdown, respiratory failure and death.

This epidemic is particularly important for nurses to be aware of as a high proportion of cases are among healthcare workers. Nurses and students can be highly vulnerable to MERS-CoV infection [9] and often, the gaps in their knowledge around infection control are severe [10]. Beyond the complexity of dealing with an increased demand for care due to the critical progression of the patients, transmission from patient-to-nurse and from patient-to patient/visitor/other staff is also of concern.

\section{Objectives}

The objectives of the study were to examine the roles of critical care nurses in Intensive Care Units (ICU) and emergency nurses, and how those roles changed during the course of the epidemic. ICU and emergency nurses were selected as they had the greatest amount of contact with infected patients. This paper reports on the role of critical care nurses and how they perceived the role to change during an epidemic and their communication competences in this international setting.

\section{Why consider roles?}

In this study, the general roles of critical care nurses were reviewed in order to compare how they changed during the epidemic. We grouped the roles into 18 categories that are applicable to most acute care nurses Table 2 .

\section{Methods}

The research team consisted of nurses who worked both at a College of Nursing in a large, public University and also in a large specialized hospital in Riyadh, Kingdom of Saudi Arabia. All team members were experienced in public health epidemics and/or critical care nursing. The questions were general and open-ended in order to elicit the responses with the least amount of surveyrelated bias.

A proposal for the research was submitted to the College of Nursing's Internal Review Board and received ethics approval to use a semi-structured questionnaire as well as written openended questions to understand the nursing roles during epidemic and non-epidemic times.

Participants signed informed consent forms. One member of the research team recruited the critical care nurses by a convenience sample of nurses who worked on wards where MERS-CoV patients were present. The inclusion criteria required that the nurses were present during the epidemic period at the hospital and also worked in an ICU or Emergency setting at the time of the increase in cases (April/May 2014). The researcher used a questionnaire that was developed by the team of nurses, after consulting the published literature. She met with each participant individually.

The nurses were asked about their role and how it changed during the recent epidemic. The participants were also asked to provide suggestions for improving nursing care in the case of future epidemic. The nurses, in the presence of a member of the research team, wrote their responses. All members of the research team read the responses. The roles that make up some of nurses' work were grouped into 18 categories and are outlined in Table 2.

Each questionnaire took between 20 and $30 \mathrm{~min}$ to complete. In total, 9 questionnaires were completed. Each questionnaire consisted of 8 demographic questions and 10 open-ended questions about nurses' roles during the epidemic period and before it. The 9 critical care nurses were from different cultural backgrounds, 2 from the Czech Republic, 2 from Malaysia, 1 each from the Philippines, Poland, UK, Pakistan, and the USA. 6 nurses had Associate Diplomas and 3 had Bachelor's degrees. The nurses ranged in age from 29-46 years old. 1 was male and 8 were female, the mean years of work experience was 13 years. All of the nurses included in the study had cared for patients during the height of the MERS-CoV outbreak in 2015.

\section{Findings}

Role of a nurse in an epidemic: example of MERS-CoV: In the initial stages of the outbreak, hospital activity carried on as usual, with little change occurring to the ER triage or admission procedures. As a result, very little disease-specific screening or isolation for patients with MERS-CoV occurred. Testing required up to 24 hours to confirm a probable case and the initial symptoms were extremely common. Over time, as more people died and healthcare workers fell ill, hospital staff began to advocate for better communication, more thorough education and procedures, specific to MERS-CoV [10]. 
Table 1: Understanding the threat presented by MERS-CoV.

\section{MERS-CoV}

A viral respiratory disease caused by a previously unidentified coronavirus

First identified in Saudi Arabia in 2012

Part of the Coronavirus family. Coronavirus can cause the common cold and also SARS

MERS-CoV symptoms: fever, cough shortness of breath, and gastrointestinal involvement

Spread by droplets, CDC (2015) continues to recommend contact and airborne precautions

Kills about $36 \%$ of those who have been diagnosed with it

Has infected healthcare workers, patients and visitors within hospital settings

Table 2: Practice activities under usual and MERS-CoV epidemic conditions.

\section{Nursing roles}

Administering medications

Assisting with elimination

Assisting with mobility

Assessing patients' health

Caring for wounds

Communication

Managing care

Delegating work

Documenting

Emotional Counselling

Patient hygiene

Making evidence based decisions

\section{Mentoring and Preceptorship}

Collecting specimens and interpreting results

Nutrition and hydration

Disease prevention and health promotion

Setting priorities

Teaching patients and families

\section{Some of the changes and challenges due to the MERS-CoV epidemic}

Most patients with symptomatic MERS-CoV experience breathing difficulties. Patients require nebulized medications and sometimes intubation- both of which increase the risk for the virus to become airborne for some time in the environment. To avoid further transmission, patients should be in negative pressure environments during nebulization and intubation

About one third of all MERS-CoV patients have nausea and diarrhea. Nursing staff responded to patients needs in a timely fashion. Infectious waste and laundry must be handled in a way to avoid further infections

Unconscious or severely weakened patients require frequent range of motion assistance. Mobile patients increase the potential for spread of the virus and require teaching, assistance with PPE and monitoring

Nurses in the emergency room increased screening for symptoms related to MERS-CoV. Patients with MERS-CoV diagnoses required continual monitoring as they could easily develop ARDS and kidney failure

Dehydration, diarrhea, the need for isolation and immobility due to general weakness all contribute to the patients' increased risk of developing wounds. Nurses increased their vigilance for wound prevention and care during the epidemic

In the Saudi environment, there are policies that are in place due to cultural considerations. As per hospital rules, it was not permitted to tell the students or family members if someone was diagnosed with MERS-CoV. In some cases, the patients were not told. This increased the risk of nosocomial spread

New units were eventually opened to specifically care for MERS-CoV patients. Prior to this, special assignments were made. Early on, cohorting of patients with probable MERS-CoV and those confirmed occurred. There could have been resulting new cases of MERS-CoV

To reduce movement in and out of rooms, and the number of people exposed to patients with MERS-CoV, nurses were required to fill in on some duties that were usually covered by housekeeping and technologists

Nurses paid special attention to the movements of tablets, papers and writing utensils that were in rooms of MERS$\mathrm{CoV}$ patients as they could act as vectors for the virus. Logs of visitors were kept and nurses monitored the process

Some nurses were themselves stressed and concerned, making it difficult for them to console concerned patients, family members and community members

In the Saudi environment, family members or a personal maid may be (under usual circumstances) involved in hygiene activities. During the epidemic, this was discouraged, further increasing the nurses' role and duties

Nurses were encouraged to check the WHO.int website, as well as the Ministry of Health often to keep up on current guidance, in relation to the epidemic. As the virus was only recently identified, little was known about how it spread or where it originated. While there was initially no official guidance on the type of respirator mask or fit testing, some nurses advocated for them, basing their demands on evidence from the published literature

First to forth year students were removed from the units and given extra nursing lab and Clinical Simulation time. Preceptorship students were left on the units with further training
previous epidemics elsewhere took on informal leadership roles

Patients were screened for the virus with nasal pharyngeal swabs as well as broncho-alveolar lavage and tracheal aspirates. Clearly nurses were required to don appropriate PPE and follow handling of specimen policies carefully. Lab staffs were also encouraged to increase precautions

Many MERS-CoV patients were dehydrated due to fever, shortness of breath and diarrhea. Hydration of patients was a priority

Patients who had MERS-CoV diagnoses and were ambulatory were reminded to wash their hands and bodies frequently, wear a mask in the presence of visitors and stay in their rooms. Designated equipment was used, where possible. Nurses and other staff were reminded to use PPE

While the patients' healing process remained the goal of the nurses and student nurses, there was also an urgency to reduce the exposure to the virus and protect patients and themselves from infection and mental stress. Consistent procedures for screening, monitoring of visitor and staff exposures, and availability of PPE needed to be prioritized Families of patients who did not have MERS-CoV were concerned about the safety of their families and required information and support. Nurses dealt with anxious family members needing information and comfort 
The role of the nurse in providing physical care: During an epidemic, all usual essential tasks must be completed for the patient, but they are frequently intensified. Having a large surge of very ill patients puts a stress on the entire health care system. Also, many of the procedures that nurses initiate (such as deep breathing and coughing, assisting with bronchoscopy, intubation/ extubation cardiopulmonary resuscitation, taking sputum samples, and suctioning) may be the method of aerosolization of coronavirus [11].

In the case of the MERS-CoV epidemic, patients with and without confirmed cases of the virus had to be carefully monitored. Nurses were providing medications to address symptoms and monitoring input and output to assess for potential renal failure, there were no effective medications available to treat MERs-CoV specifically. All confirmed and probable cases had to be cared for while nurses upheld airborne precautions.

The role of the nurse in providing emotional care: Fear is undoubtedly an unavoidable by-product of an outbreak of a deadly virus. Patients, their families and healthcare workers felt anxious when they came into contact with any person who was coughing or looking generally unwell during the time of the epidemic. Caring for fellow nurses and other colleagues who had contracted the virus added a new layer of complication and emotional stress. Nurses communicated their frustrations and concerns with one another and later with management. Nurses who had worked in previous epidemics such as SARS-CoV and H1N1 communicated their knowledge and experience with their colleagues to ensure them that this epidemic would also peak and then dissipate, if good infection control policies were followed.

The role of the nurse in teaching and communication: Of all the health professions, nurses spend the most time in face-toface conversation with patients [12]. Patients look to nurses for direction and information. While most patients in the hospital are already stressed due to the illness that brought them there, patients experienced even higher stress due to the unknown factors during the epidemic. During this epidemic, there was also a trust issue, as patients believed that information; even about their own diagnoses was being withheld. Furthermore, nurses were the main educators and resource for information with regular communication to both their families and friends about the disease. To avoid mixed messages and confusion, nurses were asked to refer patients to the Ministry of Health website, hand-outs and hotline for more information relaying consistent and up-to-date messaging.

The role of the nurse in communication through documentation: In addition to all of the regular documentation associated with patients in isolation rooms, nurses needed to log each time they entered and exited the room of a patient who was a probable or confirmed case of MERS-CoV. Likewise, nurses had to be gatekeepers of both the patient by not allowing them to wander outside of the room and their visitors. During this stressful time, some family members may be suspicious of the reasons for their names being logged.

Nurses were also required to take detailed histories related to the current illness, comorbidities and medications- but also had to start the preliminary contact tracing. The current theory regarding MERS-COV is that camels are an important reservoir for the disease. Camels are extremely important to Saudi culture with raw milk and even urine serving as drinks considered healthy and even medicinal [13].

The role of the nurse in advocating for patients and family: Advocating for patients during an epidemic is essential as communication can be difficult when they are intubated, sedated, and isolated from primary family members. Performing at least hourly vital signs including pain assessments, as patients are at high risk for sepsis. As with any sedated or intubated patient mobilization is vital to prevent bedsore and infection and turning the patient must be done at least every $2 \mathrm{~h}$.

Consistency from every nurse on every shift is also vital to keep the highest level of standards during an epidemic. Nurses must work as a team and communicate effectively in giving information to the patient and family members.

The role of the nurse in mentoring: Nurses learn to develop their professional practice by being mentored by others, often more experienced nurses. During an epidemic, nurses look to their senior nurses for communication and guidance on how to respond. Nurse leaders also tend to set the professional standards for the unit, bringing a sense of calm or panic, depending on their own feelings and comfort. Nurses who are actively preceptor students often chose to care for the most seriously ill patients themselves and leave the students to work with those who were least likely to be infectious or critical [10].

The role of the nurse in communicating disease prevention and health promotion: MERS-CoV advocacy in the Middle East pertains to keeping family visitors to minimum and completely discouraging children from coming to the hospital at all. Extended family groups are highly motivated to offer their respects to patients while they are in the hospital. Repeating answers to the same questions multiple times is necessary and demanding on the nurses' limited time. Additionally, arranging for translators to make sure the instructions are clear to help limited the spread of infection in the homes and community. Basic instructions that need consent repeating and consistency from every nurse in contact with the infected patients including: washing hands, inform other family members to come to the hospital only if they are experiencing similar symptoms, and putting on and removing all the personal protective equipment if they are insistent upon going into the infected or potential infected patient's room.

Implications for nurses: improvements for future epidemics: Critical care nurses made suggestions as to how nursing care could be improved in future epidemics. Most of the nurses would have liked to see improved general public health knowledge about the virus and what should be done about it at different levels of the health care system. One nurse wrote, "Transparency needs to be increased as it increases confidence and people know what they are dealing with. Transparency means availability of information such as what the disease is, number of people infected, even if minimal disease at the beginning of an epidemic." 
About half of the nurses mentioned a need to improve the supply chains to increase access to Personal Protective Equipment. Nurses mentioned that prevention and education needed improvement. One nurse wanted to decrease visitor traffic, especially for children. Nurses also mentioned that that there should be standardized protocols in dealing with potentially infectious patients. One nurse asked for clearer guidelines on screening and testing. Another nurse suggested that physicians should receive education on more efficient practicing in testing for MERS-CoV to avoid delay. A summary table of the lessons learned during the epidemic is included (Table 3 ).

\section{Conclusion}

Clearly the nurses' workload increased during the epidemic time, as did the stress of working with the threat of personal harm from caring for MERS-CoV patients. While many positive adaptations had been made in response to the epidemic, all nurses made suggestions for changes during future epidemics and how they can remain communication competent in an International setting. The role of the nurse during an epidemic will continue to evolve as lessons learned from each wave of the virus are implemented and new policies; procedures, supply lines, and isolation units are established.

Table 3: Lessons learned during the MERS-CoV outbreak.

Information about epidemics and disease control should be done as a part of the hospital's regular training, and reinforced during the time of outbreaks. Direction on screening, the robustness of the supply chain during a surge in demand for PPE, cohorting of patients and where to access the latest information and policies should be well-known.

Hospital management has a duty to care for their staff and to support them during times of increased workload, especially during an epidemic. Staff needs to have reassurance and adequate resources to protect their patients, themselves and their families.

Hospital management needs to give clear, responsive and timely accurate messages about the hospitals response during an epidemic, adhering to an emergency planning processes and protocols.

Nurses need to continue their development of emotional resilience and emotional labor required being a registered nurse; these skills and attributes need to be recognized and developed as part of their career pathway.

Nurses are role models in the hospitals, community and within their families. As nurses are required to maintain calm and give assurance during an epidemic, ongoing education and skill development is essential. 


\section{References}

1 Gallop (2015) Honesty/ethics in professions.

2 Morgan R (2014) Roy morgan image of professions survey 2014-nurses still most highly regarded-followed by Doctors, Pharmacists \& High Court Judges.

3 Almalki M, FitzGerald G, Clark M (2011) The nursing profession in Saudi Arabia: an overview. Int Nurs Rev 58: 304-311.

4 World Bank (2015) Nurse and midwives per 1,000 people.

5 World Health Organization (WHO) (2015) Middle East respiratory syndrome coronavirus (MERS-CoV). Summary of current situation, literature update and risk assessment.

6 Public Health Agency of Canada PHAC (2013) Interim GuidanceMiddle East respiratory syndrome coronavirus (MERS-CoV).

7 Centers for Disease Control and Prevention (2015) MERS-CoV: CDC issues updated infection control guidance for hospitals.
8 World Health Organization (2017) Middle East respiratory syndrome coronavirus (MERS-CoV).

9 Stirling BV, Harmston J, Alsobayel H (2015) An educational programme for nursing college staff and students during a MERScoronavirus outbreak in Saudi Arabia. BMC Nurs 14: 20.

10 Stirling B, Harmston J (2015) Readying nurses for clinical practice: Protecting students during an outbreak of Middle EasternCoronavirus in Saudi Arabia. Int J Educ Pract 5: 34.

11 Tran K, Cimon K, Severn M, Pessoa-Silva CL, Conly J (2012) Aerosol generating procedures and risk of transmission of acute respiratory infections to healthcare workers: a systematic review. PloS ONE 7: e35797.

12 Cohen B, Hyman S, Rosenberg L, Larson E (2012) Frequency of patient contact with health care personnel and visitors: implications for infection prevention. Jt Comm J Qual Patient Saf 38: 560-565.

13 Zumla A, Hui DS, Perlman S (2015) Middle East respiratory syndrome. Lancet 386: 995-1007. 